\title{
Functionality and Cell Senescence of CD4/ CD8-Selected CD20 CAR T Cells Manufactured Using the Automated CliniMACS Prodigy ${ }^{\circledR}$ Platform
}

\author{
Krasimira Aleksandrova ${ }^{\mathrm{a}} \quad$ Jana Leise $^{\mathrm{a}} \quad$ Christoph Priesner $^{\mathrm{a}} \quad$ Anette Melk $^{\mathrm{b}}$ \\ Fanni Kubaink $^{\mathrm{b}}$ Hinrich Abken $^{\mathrm{c} d}$ Andreas Hombach $^{c}$ Murat Aktas $^{\mathrm{e}}$ \\ Mike Essl $^{\mathrm{e}} \quad$ Iris Bürger $^{\mathrm{e}} \quad$ Andrew Kaiser ${ }^{\mathrm{e}}$ Georg Rauser ${ }^{\mathrm{e}}$ Marion Jurk ${ }^{\mathrm{e}}$ \\ Lilia Goudeva $^{f}$ Wolfgang Glienke ${ }^{g}$ Lubomir Arseniev ${ }^{a}$ Ruth Esser ${ }^{g}$ \\ Ulrike Köhla, g-i \\ ${ }^{a}$ Cellular Therapy Centre, Institute of Cellular Therapeutics, Hannover Medical School (MHH), Hanover, Germany; \\ ${ }^{b}$ Clinic for Paediatric Nephrology, Hepatology and Metabolic Disorders, Hannover Medical School (MHH), Hanover, \\ Germany; ${ }^{C}$ Center for Molecular Medicine Cologne, University of Cologne, and Dept I Internal Medicine, University \\ Hospital Cologne, Cologne, Germany; ${ }^{\mathrm{d}} \mathrm{RCl}$, Chair Gene-Immunotherapy, University Hospital Regensburg, \\ Regensburg, Germany; ${ }^{\mathrm{e}}$ Miltenyi Biotec $\mathrm{GmbH}$, Bergisch Gladbach, Germany; ${ }^{\mathrm{f}}$ Institute for Transfusion Medicine, \\ Hannover Medical School (MHH), Hanover, Germany; ${ }^{9}$ ATMP-GMP Development Unit, Institute of Cellular \\ Therapeutics, Hannover Medical School (MHH), Hanover, Germany; ${ }^{\mathrm{h}}$ Institute of Clinical Immunology, University \\ Hospital and Medical Faculty, University of Leipzig, Leipzig, Germany; ${ }^{\text {'F}}$ Fraunhofer Institute for Cell Therapy and \\ Immunology, Leipzig, Germany
}

\section{Keywords \\ Chimeric antigen receptor . GMP manufacturing of CART cells $\cdot$ CD4/CD8 selection - Telomere length · Cytotoxicity}

\begin{abstract}
Clinical studies using autologous CAR T cells have achieved spectacular remissions in refractory CD19+ B cell leukaemia, however some of the patient treatments with CAR T cells failed. Beside the heterogeneity of leukaemia, the distribution and senescence of the autologous cells from heavily pretreated patients might be further reasons for this. We performed six consecutive large-scale manufacturing processes for CD20 CAR T cells from healthy donor leukapheresis using the automated CliniMACS Prodigy ${ }^{\circledR}$ platform. Starting with a CD4/CD8-positive selection, a high purity of a median of $97 \%$ T cells with a median 65 -fold cell expansion was
\end{abstract}

achieved. Interestingly, the transduction rate was significantly higher for $\mathrm{CD} 4+$ compared to $\mathrm{CD} 8+\mathrm{T}$ cells and reached in a median of $23 \%$. CD20 CAR T cells showed a good specific IFN- $\gamma$ secretion after cocultivation with CD20+ target cells which correlated with good cytotoxic activity. Most importantly, 3 out of 5 CAR T cell products showed an increase in telomere length during the manufacturing process, while telomere length remained consistent in one and decreased in another process. In conclusion, this shows for the first time that beside heterogeneity among healthy donors, CAR T cell products also differ regarding cell senescence, even for cells manufactured in a standardised automated process.

() 2019 S. Karger AG, Basel

K. Aleksandrova and J. Leise contributed equally to this work. R. Esser and U. Köhl have shared authorship.

\section{KARGER}

(C) 2019 S. Karger AG, Basel 


\section{Introduction}

Clinical trials with CAR T cells for the treatment of malignant diseases are continuously growing rapidly, with more than 200 ongoing trials listed at clinicaltrials.gov to date [1-4]. The spectacular remissions in paediatric $B$ cell acute lymphoblastic leukaemia and adult large B cell lymphoma especially led to an expedited FDA marketing authorisation for Kymriah ${ }^{\mathrm{TM}}$ (tisagenlecleucel, Novartis) in July 2017, followed by Yescarta ${ }^{\mathrm{TM}}$ (axicabtagene ciloleucel, Kite Pharma/Gilead) in October 2017 [5].

With an increasing number of patients subjected to CAR T cell therapy, the overall manufacturing process including the quality control needs to be standardised. Although worldwide the current process is still mainly based on a complex manual manufacturing procedure observing good manufacturing practice (GMP), during recent years there has been a switch to fully automated processes, e.g., using the CliniMACS Prodigy ${ }^{\circledR}$ (Miltenyi Biotec), with successful results being demonstrated [6-11]. To date, release specifications of the final product include cell identity, vector copy number, microbiological, endotoxin, and mycoplasma testing, as well as flow cytometric quantification of transgene expression. The latter has so far been accepted as an indirect potency assay in early phase I/II trials and is partly combined with cytotoxicity assays against the respective target cells and/or quantification of cytokine secretion of the engineered $\mathrm{T}$ cells [12].

However, this does not allow the prognosis of whether an autologous CAR $\mathrm{T}$ cell product will be clinically effective in the respective patient. Reasons for a failure can be: (i) change in the surface expression of leukaemia, (ii) the heterogeneity of the cancer cells, or (iii) the reduced fitness of the source autologous cells from heavily pretreated patients. Even healthy donors show a range of biological variations regarding their immune cell distribution and cell functionality.

Therefore, it was our aim to use a standardised protocol for clinical-scale manufacturing of CD20 CAR T cells using the automated CliniMACS Prodigy ${ }^{\circledR}$ platform in order to investigate not only differences in $\mathrm{T}$ cell subpopulations and transduction efficiency, but especially cell senescence and altered functionality during the 12-day manufacturing process. For this first study we focused on leukapheresis material from healthy donors only, but this should be extended to patient material in the next step.

\section{Materials and Methods}

Manufacturing of CAR T Cells Using the Automated CliniMACS Prodigy ${ }^{\circledR}$ Platform

Peripheral blood apheresis products from 6 healthy donors (D1-D6) were obtained either from the Institute for Transfusion Medicine, Hannover Medical School (D1-D5), or from the Insti- tute for Clinical Transfusion Medicine and Immunogenetics Ulm (D6) after the donors gave their written informed consent and approval was granted by the corresponding ethics committees (No. 2830-2015 and 172/99, respectively). The processing was started within $48 \mathrm{~h}$ after the end of leukapheresis. Transport and storage of the leukapheresis was carried out at $2-14{ }^{\circ} \mathrm{C}$ (for D1-D5) or $15-25^{\circ} \mathrm{C}$ (for D6). The manufacturing of CD20 CAR T cells was performed using the automated CliniMACS Prodigy ${ }^{\circledR}$ platform representing a closed system (Miltenyi Biotec $\mathrm{GmbH}$, Bergisch Gladbach, Germany), controlled by system software version 1.0 and TCT process software version 1.2.1 (for a schematic illustration of the manufacturing process see Fig. 1). The following modifications to the manufacturing process previously described by Priesner et al. [7] were carried out. An enriched CD4+ and CD8+ cell fraction was used as the starting material instead of CD62L+ selected cells. For this purpose, GMP-grade CliniMACS ${ }^{\circledR} \mathrm{CD} 4$ and CliniMACS ${ }^{\circledR}$ CD8 (Miltenyi Biotec) reagents were used for immunomagnetic selection of the CD4+ and CD8+ cells; $1 \times 10^{8} \mathrm{~T}$ cells of the target fraction were activated by adding 1 vial $(4 \mathrm{~mL})$ of MACS GMP T cell TransAct (Miltenyi Biotec). Transduction with VSV-G pseudotyped lentiviral vector (anti-CD20 CAR LV, Miltenyi Biotec) was started approximately $24 \mathrm{~h}$ after $\mathrm{T}$ cell activation. As the human $\mathrm{AB}$ serum source, an approved medicinal product (pool-human serum P-HS/Tü, Centre for Clinical Transfusion Medicine Tübingen [ZKT], Tübingen, Germany) was used for supplementation of the cell culture medium where indicated according to the manufacturing protocol.

Sample Harvesting and Flow Cytometric Quality Control

Samples for flow cytometric analysis of cell count, cell composition, and transduction rate were taken from the leukapheresis, the target fraction after CD4/CD8 cell selection, on days 5 and 12 before and after formulation of the final product. All used materials and analytical protocols were provided by Miltenyi Biotec. If required, samples were diluted to $1 \times 10^{8}$ cells $/ \mathrm{mL}$ with CliniMACS PBS/EDTA buffer, supplemented with 0.5\% MACS BSA. The cells were labelled (10 min at room temperature in the dark) with fluorescent monoclonal antibodies $(\mathrm{mAb})$ in different combinations (CD45-VioBlue, CD4-VioGreen, CD3-FITC, CD16-PE, CD56-PE, CD20-PE-Vio770, CD14-APC, CD8-APC-Vio770) and/or a CD20 CAR detection reagent (PE) for detecting the CAR expression after transduction. 7-Amino-actinomycin-D was added to each panel to exclude dead cells from the analysis. After labelling, the samples were incubated for either $10 \mathrm{~min}$ in red blood cell lysis solution (for non-transduced or transduced cells classified as safety level 1 according to German gene engineering law) or for 20 min inside fix solution (for transduced cells classified as safety level 2 according to German gene engineering law). The samples were analysed with MACSQuant Analyser 10 and the appropriate software version.

\section{Quantification of Telomere Length}

Analytical samples (cell suspension) for examination of potential changes in the telomere length were taken at the beginning of cultivation (day 0 ) and from the final product (day 12). The samples were stored at $<-140^{\circ} \mathrm{C}$ until they were analysed. For technical and organisational reasons, no sample was drawn on day 0 of the D2 process. Due to the missing reference value from day 0 , no changes in the telomere length during the following cultivation could be analysed. Genomic DNA was extracted from CAR T cells using QIAamp DNA Mini kit (Qiagen, Hilden, Germany). DNA quantification was performed on an Infinite M200 Pro microplate reader (Tecan, Männedorf, Switzerland) using SYBR ${ }^{\circledR}$ Green I Dye (Invitrogen, Carlsbad, CA, USA). Telomere length was calculated as the abundance of telomeric template versus a single copy gene
Aleksandrova et al. 
Fig. 1. Schematic presentation of the CD20 CAR T cell manufacturing process. Process buffer, PBS/EDTA with $0.5 \%$ (w/v) human serum albumin (HSA); process medium 1 , TexMACS GMP medium supplemented with recombinant human IL-7 and IL-15, and $3 \%(\mathrm{v} / \mathrm{v})$ human AB-Serum; process medium 2, TexMACS GMP medium supplemented with IL-7 and IL-15; formulation buffer, Composol ${ }^{\circledR}$ PS with $2.5 \%(w / v)$ HSA.

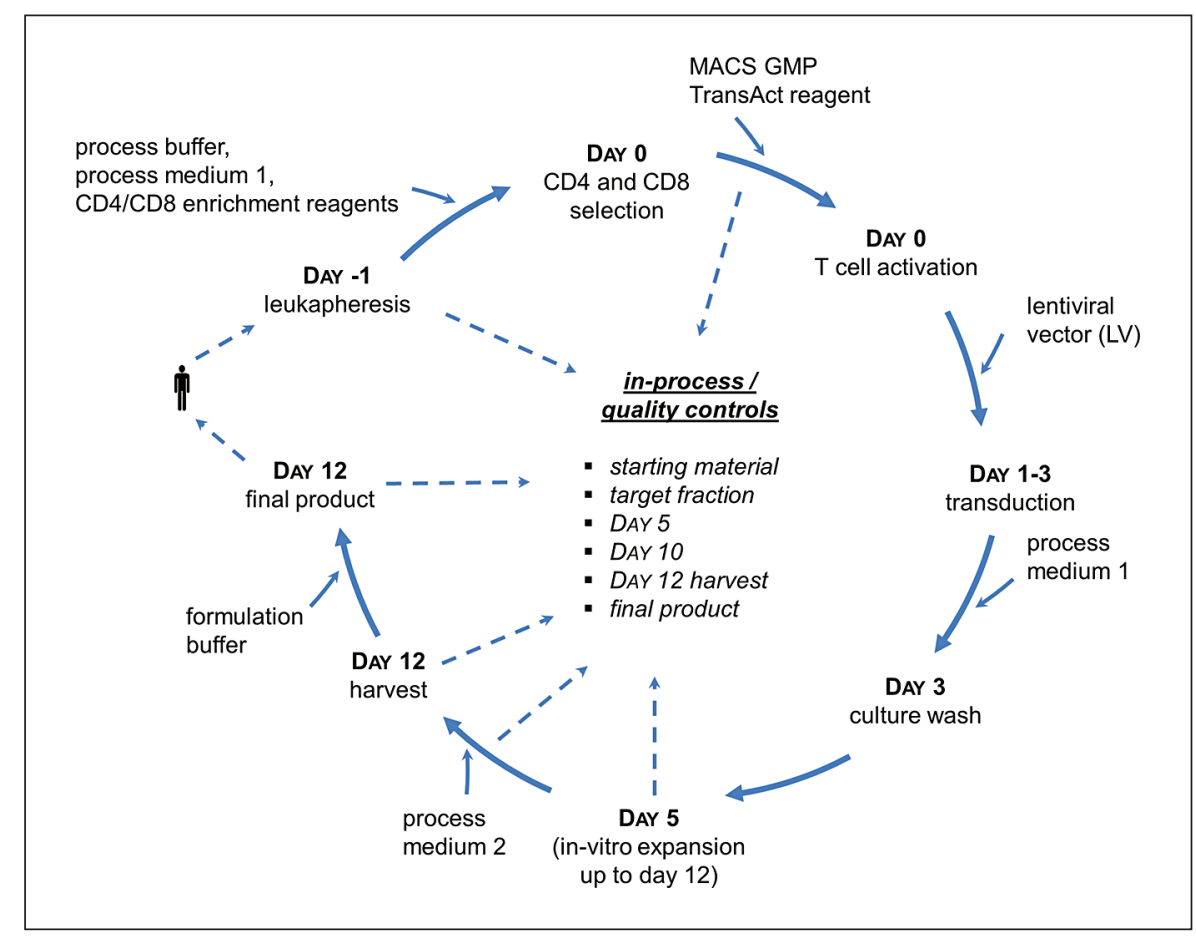

(36B4) by quantitative real-time PCR on a 7900HT fast real-time PCR system (Applied Biosystems, Darmstadt, Germany) as previously described $[13,14]$. Results are presented as the ratio (T/S) between the telomere repeat copy number $(\mathrm{T})$ to the single gene copy number (S). All measurements were performed in quadruplicates and all samples were measured on one plate to reduce interassay variability.

\section{Functional Assay}

The phenotype of CAR T cells was determined by flow cytometric analysis utilising fluorochrome-labelled $\mathrm{mAb}$ specific for CD3, CD4, CD8, CD62L, and CD45RO. Antibodies against CD3, $\mathrm{CD} 4$, and $\mathrm{CD} 8$ were purchased from Miltenyi Biotec, the anti$\mathrm{CD} 45 \mathrm{RO} \mathrm{mAb}$ was purchased from BD Biosciences (Heidelberg, Germany) and the anti-CD62L mAb was purchased from ImmunoTools GmbH (Friesoythe, Germany). Expression of the CAR was determined by staining with CD20 CAR detection reagent (PE) from Miltenyi Biotec. Cells were analysed using a Canto II cytometer (BD Biosciences) equipped with the Diva software.

CAR T cells $\left(0.5-4 \times 10^{4}\right.$ cells/well) from D5 and D6 were cocultivated for $48 \mathrm{~h}$ in 96-well round-bottom plates with CD20transfected or non-modified HEK293T cells (each 2.5-5 $\times 10^{4}$ cells/well). Specific cytotoxicity of CAR T cells against antigenpositive target cells was monitored by an XTT-based colorimetric assay [15] using a Cell Proliferation Kit II (Roche Diagnostics, Mannheim, Germany). The viability of tumour cells was calculated as the mean values of six wells containing tumour cells subtracted by the mean background level of wells containing medium only. Non-specific formation of formazan due to the presence of $\mathrm{T}$ cells was determined from triplicate wells containing $\mathrm{T}$ cells in the same number as in the corresponding experimental wells. The number of viable tumour cells in experimental wells was calculated as follows: viability $(\%)=[\mathrm{OD}$ (experimental wells - corresponding number of T cells)]/[OD (tumour cells without T cells - medium)] $\times 100$. Cytotoxicity (\%) was defined as $100-$ viability (\%). Supernatants were tested for IFN- $\gamma$ by ELISA utilising solid-phase bound anti-IFN- $\gamma$ capture antibody $(1 \mu \mathrm{g} / \mathrm{mL})$ and detection by the bio- tinylated anti-IFN- $\gamma$ detection antibody $(0.5 \mu \mathrm{g} / \mathrm{mL}$; both BD Biosciences). The reaction product was visualised by a peroxidasestreptavidin conjugate $(1: 10,000)$ and ABTS (Roche Diagnostics).

\section{Microbiological, Endotoxin, and Mycoplasma Testing}

Microbiological examination according to Ph. Eur. 2.6.27 was performed for three processes (D4-D6) with sampling of the starting material (apheresis), the cell culture on day 10, and the final cell product on day 12 . The analysis was performed by the Institute for Medicinal Microbiology and Hospital Epidemiology (MHH, Hannover, Germany). Samples for endotoxin control were taken and tested according to Ph. Eur. 2.6.14 on day 5 and from the final product of five manufacturing runs using Nexgen Endosafe ${ }^{\circledR}$ PTS device (Charles River Laboratories, Charleston, SC, USA). Mycoplasma testing was performed according to Ph. Eur. 2.6.7 on three final cell products by a contracted analytical company (Minerva Analytix, Berlin, Germany).

\section{Statistics}

Statistical significance and $p$ values were calculated by multiple $t$ test (Holm-Sidak method, with $\alpha=0.05)$ using GraphPad Prism 7.

\section{Results}

\section{CD4/CD8 Selection}

The proportion of CD3+ $\mathrm{T}$ cells in the six apheresis products (D1-D6) varied between 38 and $58 \%$ of the leucocytes, which was in the expected range for healthy donors. According to the starting specification of $1 \times 10^{9}$ labelled T cells (CD4+ and CD8+), a median of $2.2 \times 10^{9}$ leucocytes (range 1.8-2.8 $\times 10^{9}$ ), and apheresis volume of 20-65 mL were needed for initiating the automated CD4/ CD8-positive cell selection. 
A

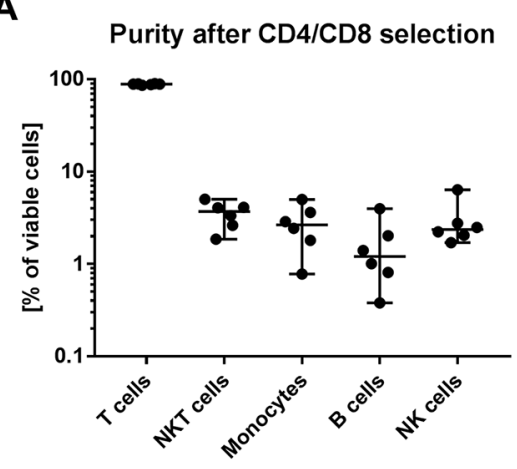

C

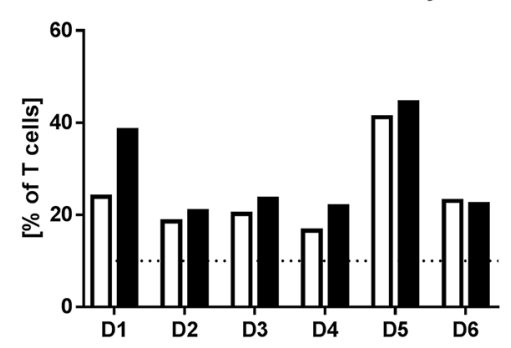

B

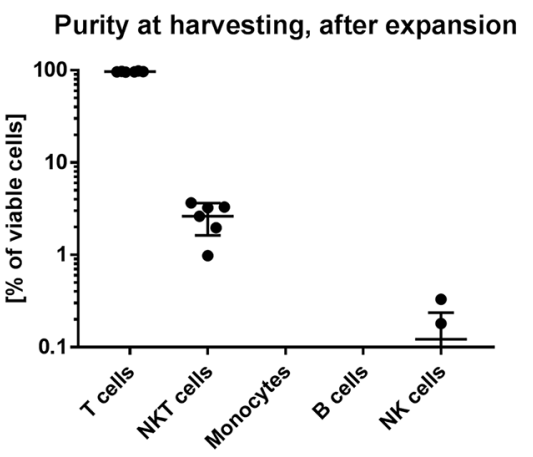

D Transduction efficiency CD4 and CD8

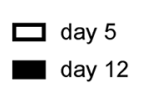

$\square \operatorname{CD} 4$ $\square \operatorname{CD8}$
Fig. 2. Purity and transduction efficiency. a Proportions of the main cell subtypes in the target fraction after CD4 and CD8 immunomagnetic selection (median, range of 6 donors). b Cellular composition at the end of the CD20 CAR T cells manufacturing process (median, range of 6 donors). c Proportion of transduced

The median recovery of the $\mathrm{T}$ cells postselection was $68 \%$ (range $60-71$ ), whereas $73 \%$ (range $63-77$ ) of the CD4+ and 65\% (range 53-70\%) of the CD8+ cells were harvested. The achieved median purity of $\mathrm{T}$ cells in the selected fraction was $89 \%$ (range $86-90$ ), the contaminating cell subpopulations were determined as 3.7\% NKT cells, $2.7 \%$ monocytes, $2.4 \% \mathrm{NK}$ cells, and $1.2 \%$ B cells (median, $n=6$; Fig. 2a). Of the selected fraction, $14 \%$ (range 13-15; corresponding to approx. $1 \times 10^{8} \mathrm{~T}$ cells) were further processed.

\section{Cell Transduction}

Three different lentiviral vector batches were used for cell transduction on day 1 of the manufacturing processing, resulting in a multiplicity of infection (MOI) of 26 for D1, MOI of 75 for D2, D3, D4, and D6, and MOI of 57 for D5. The median transduction efficiency was $22 \%$ on day 5 (range 17-41) and $23 \%$ in the final product (range 2145; Fig. 2c). Transduction efficiency was significantly higher $(p<0.05)$ for CD4+ compared to CD8+ T cells (median 26\%, range 20-51) for CD4 and 14\% (range 8-23) for CD8 on day 5, median 30\% (range 25-55) for CD4 and 17\% (range 11-29) for CD8 in the final product (Fig. 2d).
CD20 CAR+ T cells among all T cells (single values of 6 donors). d Transduction efficiency among CD4+ and CD8+ T cells on day 5 of the cultivation and in the final product ( $n=6$ donors; the line in the box represents the median, the whiskers extend from the minimal to maximal value).

\section{Cell Expansion and Composition}

A consistent cell expansion during the automated cell cultivation period resulted in a robust, 65 -fold (range 46to 81 -fold) median increase of the $\mathrm{T}$ cell count during the 12-day manufacturing process (Fig. 3a). Accordingly, a considerable amount of $\mathrm{T}$ cells was harvested at the end of all production processes - the median yield was $1.5 \times$ $10^{9}$ (range $\left.1.1-3.0 \times 10^{9}\right)$ CAR+ T cells out of $6.3 \times 10^{9}$ (range $4.3-7.6 \times 10^{9}$ ) viable $\mathrm{T}$ cells (Fig. $3 \mathrm{~b}$ ). The viability of the cell suspension was $>95 \%$ at the final harvest of all processes.

With a median purity of $97 \%$ T cells (range 96-99), the final product contained some impurities of NKT and NK cells: median 2.9\% (range 1.0-3.7) and 0.07\% (range $0.04-0.3$ ), respectively (Fig. 2 b). No other cell subpopulations could be detected at measurable amounts.

The proportion of CD4+ and CD8+ T cells changed in a comparable manner during the cell cultivating starting with a median postselection CD4/CD8 ratio of 2.4 (range $1.8-3.1$ ), decreasing to 1.9 (range $1.1-2.8$ ) on day 5 and 1.7 (range 1.1-2.3) in the final product (Fig. 3c, d). The observed decrease of the $\mathrm{CD} 4 / \mathrm{CD} 8$ ratio reflects the significant larger expansion of the CD8+ cells compared to the CD4+ T cells: median CD8+ cell expansion 79-fold 
A

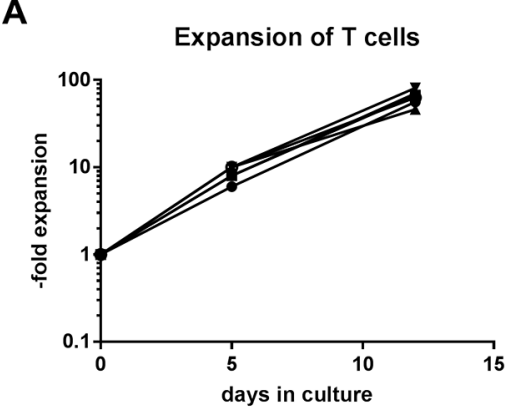

C

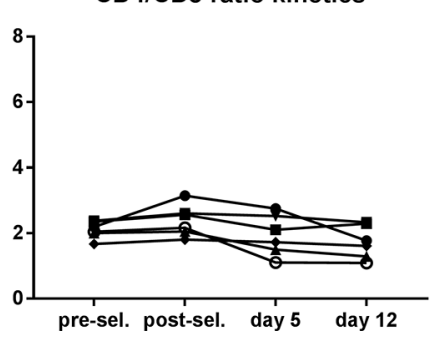

B

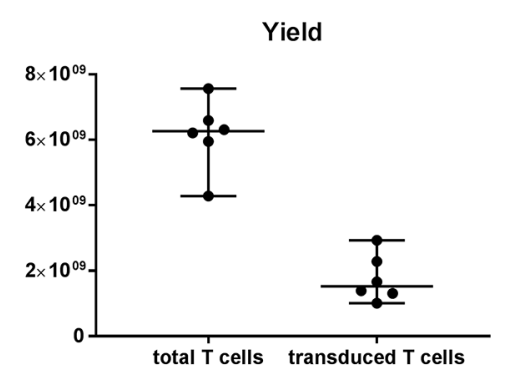

D
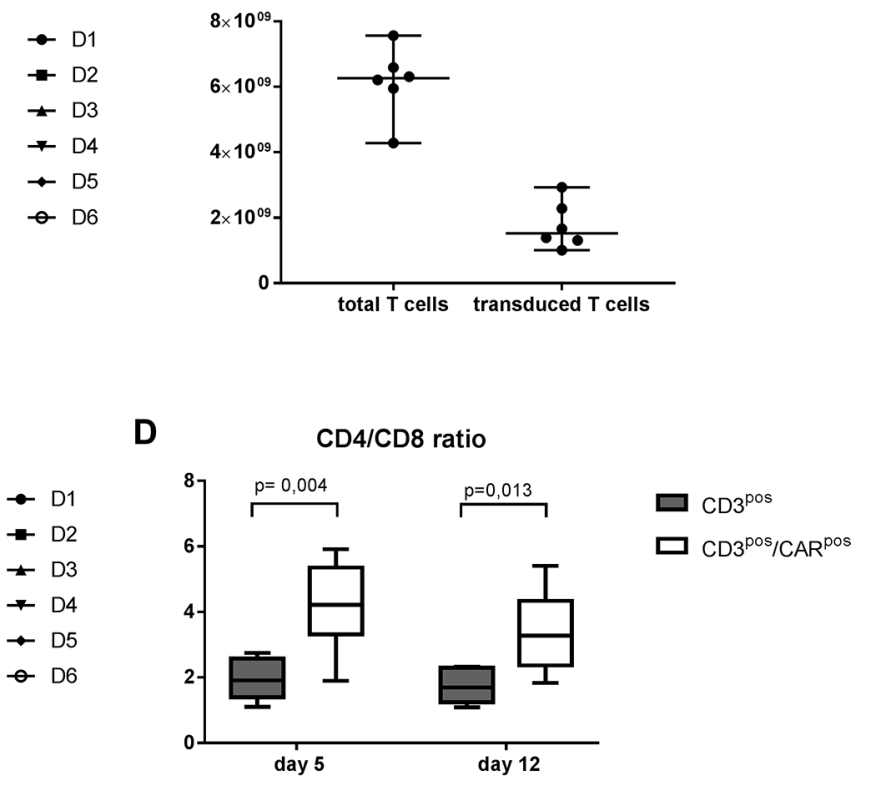

Fig. 3. Expansion, yield, and cellular distribution during cell cultivation. a T cell expansion during the manufacturing process. $\mathbf{b} \mathrm{T}$ cell and CAR+ T cell counts in the final product (median, range of 6 donors). $\mathbf{c}$ Changes in the CD4/CD8 ratio within the T cells during the expansion phase. $\mathbf{d}$ Comparison of the CD4/CD8 ratio among total and transduced CAR $+\mathrm{T}$ cells $(n=6$ donors; the line in the box represents the median, the whiskers extend from the minimal to maximal value).

(range 61- to 95-fold) compared to a median CD4+ cell expansion of 56 -fold (range 38 - to 80 -fold; $p=0.023$ ).

A significantly higher CD4/CD8 ratio $(p<0.05)$ was calculated when analysing only the CAR+ T cell population on both day 5 and day 12 for all processes: median 4.2 (range 1.9-5.9) on day 5, and 3.3 (range 1.8-5.4) on day 12 (Fig. 3d). At harvesting, a median of $75 \%$ of the CAR+ T cells (range 69-84) were CD4+ and 22\% (range 16-27) were CD8+.

\section{Telomere Length for Extended Product \\ Characterisation}

For further product characterisation concerning the cell senescence after expansion, the telomere length was analysed at the start of culture (day 0) and in the final product (day 12). In three out of five production processes (D1, D3, and D4), cells of the final product showed an increase in telomere length compared to cells before expansion, whereas the telomere length remained consistent in one process (D5), and decreased in another process (D6), as shown in Figure 4 as the T/S ratio before and after expansion.

Cytotoxicity of CAR T Cells against Leukemic Targets

Prior to functional testing, the processed cells were phenotypically characterised by flow cytometry. $\mathrm{T}$ cells

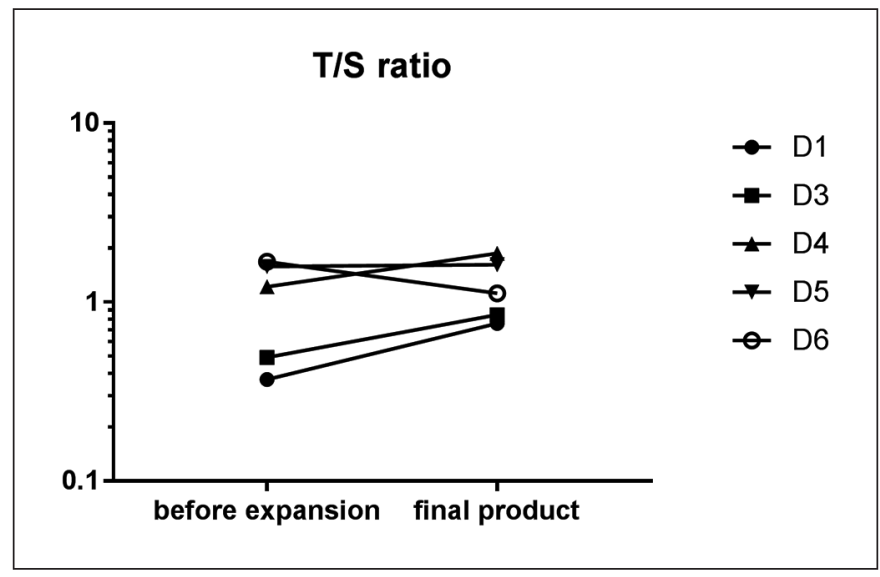

Fig. 4. Telomere length. Ratio (T/S) between the telomere repeat copy number (T) to the single gene copy number (S) of CD20 CAR $\mathrm{T}$ cells at the start of cultivation (before expansion) and after expansion (final product). Data of 5 out of 6 processes (D1, D3-D6) are shown.

showed a predominantly (about $80 \%$ ) naïve CD62L+ CD45RO- phenotype. After $48 \mathrm{~h}$ of cocultivation with HEK293T cells, the CD20 CAR T cells secreted IFN- $\gamma$ in a dose-dependent manner in the presence of transfected CD20+ HEK293T cells, but not in the presence of CD20- HEK293T cells (Fig. 5a, b). No IFN- $\gamma$ release 


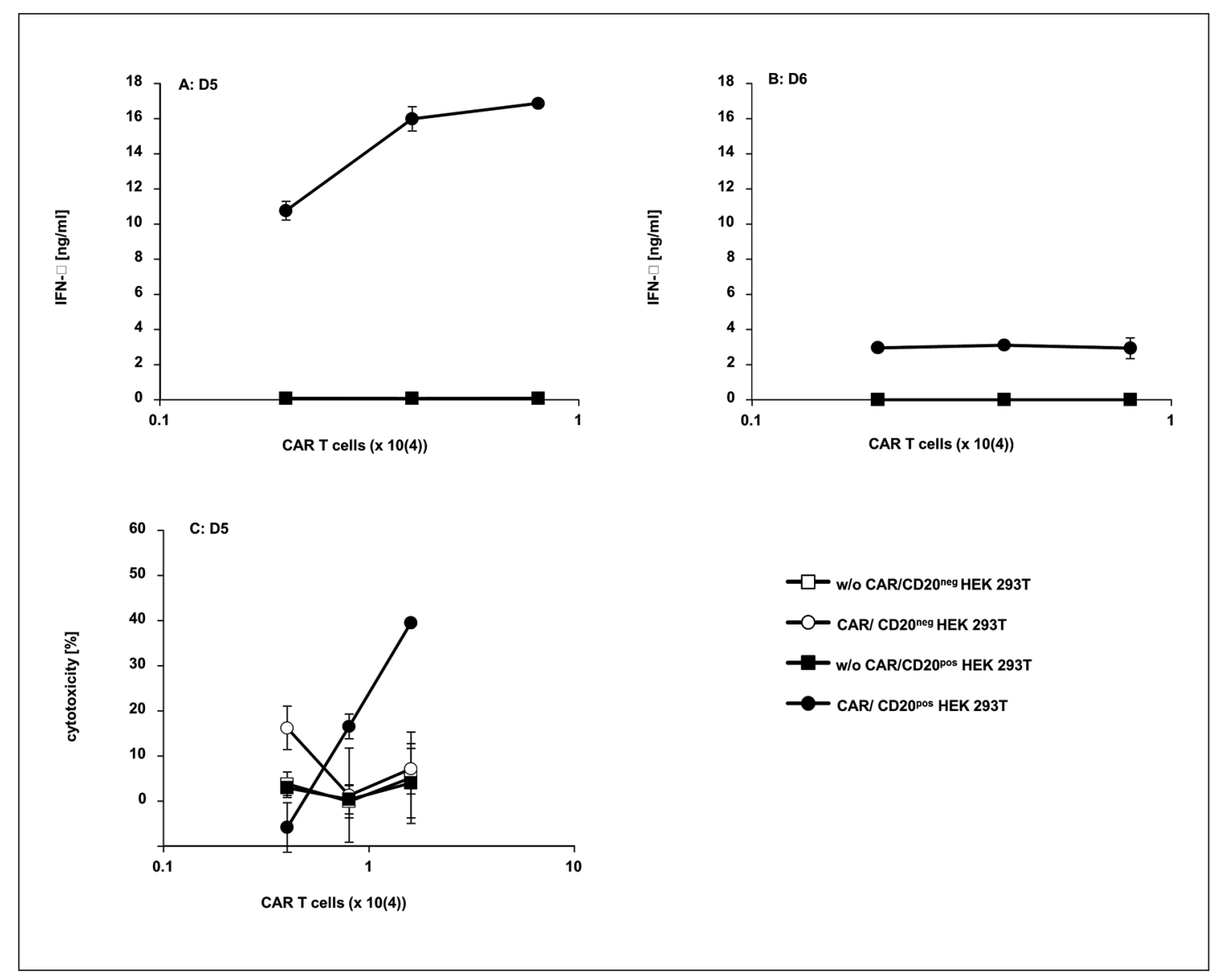

Fig. 5. Antigen-specific activation of CAR T cells. CD20 CAR T cells and non-modified T cells (w/o CAR) as the controls (each $0.2-1.6 \times 10^{4}$ cells/well) of donor D5 (a, c) and donor D6 (b), respectively, were cocultivated for $48 \mathrm{~h}$ with HEK293T cells with or without transgenic CD20 (each $2.5 \times 10^{4}$ cells/well) in 96-well round-bottom plates. b, c Supernatants were harvested and the IFN- $\gamma$ concentration was determined by ELISA. $\mathbf{c}$ The viability of tumour cells was determined by a tetrazolium salt-based XTT assay and cytotoxicity was determined as described in Materials and Methods. Values represent the mean of triplicates \pm SD.

was induced when non-transduced autologous (D6) or allogeneic T cells (D5) were cocultivated with CD20+ HEK293T cells demonstrating the CAR-specific dependency of T cell activation. CAR T cells were also cytotoxic against CD20+ target cells but not against CD20-HEK293T cells. In contrast, T cells without CAR were not cytotoxic against CD20+ or CD20- HEK293T cells (Fig. 5c).

\section{Microbiological Examination and Measurements of \\ Endotoxin and Mycoplasma}

Microbiological examination did not show microbial growth in any of the tested samples. For endotoxin testing, all results were below the quantification limit of $0.5 \mathrm{EU} / \mathrm{mL}$ and no mycoplasma contamination was detected.

\section{Discussion}

The CliniMACS Prodigy ${ }^{\circledR}$ system is currently the most widely used automated platform for the manufacturing of CAR T cells [9], achieving successful results as shown previously $[6-8,10,11]$. The ability to conduct all manufacturing steps needed for gene modification of designated target cells, i.e., the CD4/CD8 cell immunomagnetic selection, T cell activation, transduction, expansion, and formulation of the final product in a sterile single-use tubing set (TS520) using GMP-grade reagents in a closed system, enables safe manufacturing of clinical-grade cell therapeutics according to GMP regulations. The current study using the automated process includes both: (i) application of a standardised manufacturing protocol to reach highly pure $\mathrm{CD} 3+\mathrm{CAR} \mathrm{T}$ cells, including CAR-transduced CD4+ and CD8+ subpopulations, and most importantly (ii) functionally active, "healthy" CAR T cells. 
The results of all processing steps were comparable and in a predicted tight range, which fulfils one of the main prerequisites for manufacturing according to GMP. The median cell recovery after immunomagnetic selection $(68 \%)$ and the high purity of the target fraction $(89 \% \mathrm{~T}$ cells) allows standardisation of the starting conditions for the subsequent cell engineering and expansion. Some contaminating other cell types, mainly NKT, NK cells, some B cells, and monocytes, were reduced during the following cultivation in the T cell-adapted culture medium. As only a small amount of the CD4/CD8 enriched target cell fraction is used for the lentiviral vector-transduction and cell expansion, the remaining enriched fraction can be cryopreserved and used later for additional manufacturing processes if necessary, as already shown [7]. The T cell activating reagent used (MACS GMP T cell TransAct), with biodegradable colloidal nanomatrix particles and covalently bound CD3/CD28, combines the advantages of efficient $\mathrm{T}$ cell stimulation with no need for additional removal steps. The MACS GMP T cell TransAct is cleared during the regular culture washing for removing the overspill of lentiviral vector on the third day of cultivation.

The $\mathrm{T}$ cell activation with MACS GMP T cell TransAct and the further cultivation in supplemented culture medium (TexMACS GMP Medium plus IL-7 and IL-15, as well as human $A B$ serum up to process day 5) achieved robust and meaningful (65-fold) expansion of the T cells and a high final CD $3+\mathrm{T}$ cell purity of $\geq 96 \%$. Both results are in line with previously published data from similar manufacturing processing with the CliniMACS Prodigy ${ }^{\circledR}$ system $[8,11]$. This reflects an improvement of manufacturing process based on CD62L-selected $\mathrm{T}$ cells where a wider range of $82-99 \%$ CD3 + T cells was reported [7]. Additionally, the transduction efficiency of a median of $23 \%$ of the overall $\mathrm{T}$ cells in the present study was sufficient to produce relevant amounts of target cells in the clinical product. The expansion rate of CD8+ T cells (median 79-fold) was significantly higher than the expansion rate of CD4+ T cells (median 56-fold, $p=0.023$ ). As a result, the ratio of $\mathrm{CD} 4 / \mathrm{CD} 8$ decreased during the cultivation period (from 2.4 to 1.7), but still at no time point was it $<1$ in contrast to previous data [7]. Interestingly, the transduction efficiency was significantly higher $(p<0.05)$ for CD4+ compared to CD8+ T cells, in a median of 30 versus $17 \%$, respectively. Similar findings have been reported by other authors; however, the preferable transduction efficiency for CD4+ in comparison to CD8+ cells was not statistically significant $[16,10]$.

The fitness and proliferative capacity of $\mathrm{T}$ cells differs between donors, and especially in patients who are heavily pretreated. Quantification of the telomere length is a well-established method to detect cell senescence $[13,14]$. It could be shown previously that a longer donor leukocyte telomere length is associated with improved survival after haematopoietic cell transplantation, e.g., in severe aplastic anaemia $[17,18]$. In this respect, telomere shortening is associated with genomic instability and results in cell senescence or apoptosis. To date, no data exist about the senescence of leukocytes and especially $\mathrm{T}$ cells/CAR T cells during manufacturing using the CliniMACS Prodigy ${ }^{\circledR}$ platform.

In the present study, cell senescence during the 12-day expansion period was investigated and a varied picture was found. While T cells/CAR T cells showed an increase in telomere length in 3 out of 5 products during the overall manufacturing process, the telomere length remained consistent in one and decreased in another processed $\mathrm{T}$ cell population. This reflects the broad range of biological differences even among healthy donors. Till et al. [19] reported a shortened telomere length after ex vivo expansion of CD3-selected T cells. This different observation could be due to differences in the manufacturing protocol (especially the T cell activation scheme) as well as the usage of patient material (lymphoma patients) for gene engineering and cell expansion in contrast to healthy donors' collective analysis in the present work. The observed elongation of the telomere length after cell expansion could also be explained by a natural positive selection of the fittest cells during the cell cultivation.

The IFN- $\gamma$ release after cocultivation with target cells and cytotoxicity assays clearly showed functional CAR T cells, although even among healthy donors differences in the cytokine secretion and the correlating lytic activity against a CD20+ malignant cell line were observed.

\section{Conclusions}

These first experiments focusing on the analysis of phenotypic changes as well as functionality and senescence of CAR T cells manufactured using the CliniMACS Prodigy ${ }^{\circledR}$ platform indicated that further detailed experiments concerning $\mathrm{T}$ cell metabolism during transduction and cell expansion are necessary. During cell processing there might be a selection pressure for the survival of the fittest CAR T cells as we could show for the majority of the CAR T cell products. However, our preliminary results have to be confirmed by a broader number of donor products and most importantly studies have to be conducted with starting material of heavily pretreated patients as well.

\section{Acknowledgements}

This project was supported by the German Federal Ministry of Education and Research mainly with ref. No. FKZ 01EK1507B, but also in part with ref. No. 01EO0802 (Integrated Research and Treatment Center Transplantation). 


\section{Statement of Ethics}

For this study donors gave their written informed consent and approval was granted by the corresponding ethics committees (No. 2830-2015 and 172/99, respectively).

\section{Disclosure Statement}

M. Aktas, M.E., I.B., A.K., G.R., and M.J. are employees of Miltenyi Biotec $\mathrm{GmbH}$. No competing financial interests exist for the remaining authors.

\section{References}

1 Holzinger A, Barden M, Abken $\mathrm{H}$. The growing world of CAR T cell trials: a systematic review. Cancer Immunol Immunother. 2016 Dec;65(12):1433-50.

2 Hartmann J, Schüßler-Lenz M, Bondanza A, Buchholz CJ. Clinical development of CAR T cells-challenges and opportunities in translating innovative treatment concepts. EMBO Mol Med. 2017 Sep;9(9):1183-97.

3 Salmikangas P, Kinsella N, Chamberlain P. Chimeric Antigen Receptor T-Cells (CAR TCells) for Cancer Immunotherapy - Moving Target for Industry? Pharm Res. 2018 May; 35(8):152.

4 Abken H. Driving CARs on the Highway to Solid Cancer: Some Considerations on the Adoptive Therapy with CAR T Cells. Hum Gene Ther. 2017 Nov;28(11):1047-60.

5 Maude SL, Laetsch TW, Buechner J, Rives S, Boyer M, Bittencourt $\mathrm{H}$, et al. Tisagenlecleucel in Children and Young Adults with B-Cell Lymphoblastic Leukemia. N Engl J Med. 2018 Feb;378(5):439-48.

6 Kaiser AD, Assenmacher M, Schröder B, Meyer M, Orentas R, Bethke U, et al. Towards a commercial process for the manufacture of genetically modified T cells for therapy. Cancer Gene Ther. 2015 Mar;22(2):72-8.

7 Priesner C, Aleksandrova K, Esser R, MockelTenbrinck N, Leise J, Drechsel K, et al. Automated enrichment, transduction and expansion of clinical-scale CD62L+ T cells for manufacturing of GTMPs. Hum Gene Ther. 2016 Oct;27(10):860-9.
8 Mock U, Nickolay L, Philip B, Cheung GW, Zhan H, Johnston IC, et al. Automated manufacturing of chimeric antigen receptor $\mathrm{T}$ cells for adoptive immunotherapy using CliniMACS prodigy. Cytotherapy. 2016 Aug; 18(8):1002-11

9 Gee AP. GMP CAR-T cell production. Best Pract Res Clin Haematol. 2018 Jun;31(2): 126-34.

10 Zhu F, Shah N, Xu H, Schneider D, Orentas $\mathrm{R}$, Dropulic B, et al. Closed-system manufacturing of CD19 and dual-targeted CD20/19 chimeric antigen receptor $\mathrm{T}$ cells using the CliniMACS Prodigy device at an academic medical center. Cytotherapy. 2018 Mar;20(3): 394-406.

11 Lock D, Mockel-Tenbrinck N, Drechsel K, Barth C, Mauer D, Schaser T, et al. Automated Manufacturing of Potent CD20-Directed Chimeric Antigen Receptor T Cells for Clinical Use. Hum Gene Ther. 2017 Oct;28(10): 914-25.

12 Köhl U, Arsenieva S, Holzinger A, Abken H. CAR T Cells in Trials: Recent Achievements and Challenges that Remain in the Production of Modified T Cells for Clinical Applications. Hum Gene Ther. 2018 May;29(5):55968.

13 Cawthon RM. Telomere measurement by quantitative PCR. Nucleic Acids Res. 2002 May;30(10):e47.
14 Melk A, Tegtbur U, Hilfiker-Kleiner D, Eberhard J, Saretzki G, Eulert C, et al. Improvement of biological age by physical activity. Int J Cardiol. 2014 Oct;176(3):1187-9.

15 Jost LM, Kirkwood JM, Whiteside TL. Improved short- and long-term XTT-based colorimetric cellular cytotoxicity assay for melanoma and other tumor cells. J Immunol Methods. 1992 Mar;147(2):153-65.

16 Blaeschke F, Stenger D, Kaeuferle T, Willier S, Lotfi R, Kaiser AD, et al. Induction of a central memory and stem cell memory phenotype in functionally active CD4+ and CD8+ CAR T cells produced in an automated good manufacturing practice system for the treatment of CD19+ acute lymphoblastic leukemia. Cancer Immunol Immunother. 2018 Jul;67(7): 1053-66.

17 Gadalla SM, Aubert G, Wang T, Haagenson M, Spellman SR, Wang L, et al. Donor telomere length and causes of death after unrelated hematopoietic cell transplantation in patients with marrow failure. Blood. 2018 May;131(21):2393-8.

18 Wang C, Zhang T, Wang Y, Li Y, Liu C, Liu $\mathrm{H}$, et al. The shortening telomere length of $\mathrm{T}$ lymphocytes maybe associated with hyper-function in servere aplastic anemia. Mol Med Rep. 2018 Jan;17(1):1015-21.

19 Till BG, Jensen MC, Wang J, Qian X, Gopal AK, Maloney DG, et al. CD20-specific adoptive immunotherapy for lymphoma using a chimeric antigen receptor with both CD28 and 4-1BB domains: pilot clinical trial results. Blood. 2012 Apr;119(17):3940-50. 